\title{
Circuit
}

Musiques contemporaines

\section{Phrases musicales}

\section{La musique dans la philosophie de Wittgenstein}

Musical Phrases

\section{The Function of Music in the Philosophy of Wittgenstein}

\section{Antonia Soulez}

Volume 17, numéro 1, 2007

Le génome musical

URI : https://id.erudit.org/iderudit/016772ar

DOI : https://doi.org/10.7202/016772ar

Aller au sommaire du numéro

Éditeur(s)

Les Presses de l'Université de Montréal

ISSN

1183-1693 (imprimé)

1488-9692 (numérique)

Découvrir la revue

\section{Citer cet article}

Soulez, A. (2007). Phrases musicales : la musique dans la philosophie de Wittgenstein. Circuit, 17(1), 27-47. https://doi.org/10.7202/016772ar

\section{Résumé de l'article}

L'auteure examine ici la portée du paradigme musical dans la philosophie de Wittgenstein. Le cadre de cet examen est l'approche à partir de Wittgenstein de ce que les philosophes ont aujourd'hui à dire de la signification musicale. Deux styles d'approche sont suggérés, l'un projectif bien marqué par l'idée de " musikalische Gedanke » dans le Tractatus et révélateur d'une filiation à laquelle appartient Wittgenstein, et l'autre horizontal suivant l'axe grammatical du rapport esthétique d'appropriation du geste de l'oeuvre à travers une écoute active. Cette conception intègre une exploration de la résonance des aspects, dans une troisième phase qui ne sera pas développée. Comment Wittgenstein remplit-il certains critères d'appartenance à ce que Lydia Goehr appelle « la quête formelle de l'autonomie du musical » est une question qui conduit l'auteure à re-situer le nouage musique et société chez Wittgenstein, par contraste avec Adorno, en mettant en relief les conditions auxquelles une compréhension interne d'un " contenu formel " (Granger) peut se combiner ici avec le contexte d'une pratique.

Soulez met donc l'autonomie musicale selon Wittgenstein à l'épreuve du contexte des formes de vie, dans une phase où dominent les « jeux » d'une grammaire comparative motivée par l'analogie de la compréhension d'une phrase du langage avec celle de la compréhension d'une phrase musicale. Mettant l'accent sur le caractère actif de la compréhension, doublée d'une performance, l'auteure entend montrer comment Wittgenstein pense la musique avec la société autrement qu'Adorno, donc sans référence dialectique. La clef est une connexion inédite entre immanentisme de la signification musicale à l'échelle d'une phrase, comme le pense Wittgenstein et ses contemporains de l'École de Vienne, et un schéma esthético-pragmatique de " context-dependency ". De là s'ensuit une forme d'autonomie compatible avec la sphère extramusicale. $\mathrm{Si}$ " tout un monde se tient dans une phrase musicale ", comme le dit Wittgenstein, c'est bien au sens où un motif répercute certains aspects d'un " monde " auquel nous contribuons en le " faisant ».
Ce document est protégé par la loi sur le droit d'auteur. L'utilisation des services d'Érudit (y compris la reproduction) est assujettie à sa politique d'utilisation que vous pouvez consulter en ligne.

https://apropos.erudit.org/fr/usagers/politique-dutilisation/ 


\title{
Phrases musicales
}

\section{La musique dans la philosophie de Wittgenstein}

\author{
Antonia Soulez
}

\section{Introduction}

Wittgenstein accorde à la musique une place prépondérante. On pourrait dire qu'avec lui, dans sa philosophie, la musique est partout, explicitement, implicitement. Elle habite son écriture, sa philosophie comme elle a habité sa vie dans l'intimité mais aussi dans les cercles plus éloignés de sa vie privée, à commencer par ceux qui se formaient autour de sa propre famille qui débordaient évidemment le cadre étroitement familial ${ }^{1}$. Certains parlent à son propos d'une philosophie musicale. La musique qu'il aimait, du répertoire romantique, mais aussi la musique dont il ne parle pas, celle de ses contemporains de l'École de Vienne, et encore la musique qu'il déclare ne pas aimer, notamment Mahler, toutes ces musiques, tous ces styles sont présents dans son œuvre par un mode de présence qui même négatif ne laisse pas de frapper, d'impressionner le lecteur, et n'est en tout cas jamais anodin. Lui-même clarinettiste, il pratiquait la musique. Il pouvait lors d'un concert de musique de chambre faire part de son interprétation et montrer comment il fallait jouer telle partie, en prenant en quelque sorte virtuellement la baguette du chef d'orchestre. Le jeu d'exécution avait à ses yeux une importance très spéciale et peut-être ne serait-il pas exagéré d'y voir contenue toute une philosophie de l'interprétation associée au geste et à la physionomie de celui qui comprend et manifeste sa compréhension d'une manière qui fait partie du jeu en question, et où par conséquent comprendre et produire une compréhension comme on exécute une pièce musicale sont absolument solidaires.
1. Voir à ce sujet la correspondance entre Wittgenstein et Rudolph Koder (Wittgenstein et Koder, 2002), qui contient également deux essais par Martin Alber sur les aspects musicaux de la vie et l'œuvre de Wittgenstein. 
Les philosophes qui se penchent sur l'importance que la musique représente aux yeux de Wittgenstein distinguent en général deux fonctions : la fonction de modèle ou paradigme dans le Tractatus logico-philosophicus. C'est pour définir celle-ci par rapport à la méthode de projection que Wittgenstein invoque la musique dans une série de propositions. Cette approche projective est centrale dans la méthode de Wittgenstein. Traduite dans les termes de l'architecture, elle a pu inspirer Thomas Bernhard dans son roman Corrections, mais également nombre de travaux sur les rapports entre la maison que Wittgenstein a construite pour sa sœur Margarete à Vienne dans les années 1920 et l'architecture logique de son traité. Sous cet aspect architecturologique, la musique tombe en effet aisément sous le genre des arts constructifs.

La seconde fonction est celle de l'analogie pour saisir comment on comprend une phrase dans la philosophie seconde. Le rôle que joue le paradigme de l'œuvre d'art, notamment la musique, dans le cadre de la sémantique de la phrase retient en particulier l'attention. Au regard de la question du sens, de sa complétude, du raffinement de la forme et de son caractère structuralement achevé, le paradigme de la musique associé à celui de l'architecture quand il s'agit d'apprécier l'expressivité de la forme, ou de juger du caractère «ajusté » des formes d'un bâtiment à l'objectif qu'il sert, joue à différents niveaux qui méritent d'être examinés.

Par ailleurs, je vois quant à moi une troisième fonction généralement passée sous silence, soit parce que la dernière philosophie n'est pas encore bien connue, soit parce que les connaisseurs n'y voient le plus souvent rien d'artistique. C'est la philosophie des aspects. La musique remplit à cet égard, selon moi, la fonction d'un paradigme de résonance qui d'ailleurs n'est pas complètement sans précédent. Elle réactive d'après moi des éléments d'une tradition axée sur les qualités secondes, les qualia, reprise en compte dans l'approche psycho-physiologique, par exemple celle de Helmholtz, et qui refait surface dans la Structure de l'apparence de Goodman. Sous l'angle des qualia, en effet, la musique est interpellée sur le plan de la réception esthétique de l'auditeur apte, dit Wittgenstein, à exprimer ce qu'il comprend et à le jouer de façon correspondante. Les connexions avec les développements sur l'harmonie méritent également toute l'attention. Il faudrait pour les saisir une investigation à plusieurs registres nouant ensemble quoique distinctement théories des formes d'audition, jeux de langage conceptuels afférant à la psychologie de l'audition et de la compréhension de ce qui est entendu, et approches scientifiques de phénomènes psycho-physiologiques et acoustiques. Les correspondances sémantiques entre ces trois registres sont nombreuses et significatives. Elles dessinent des 
font philosophes, les philosophes discutent les thèses scientifiques, les scientifiques s'adressent aux artistes aussi. La raison à cela est l'inscription parfois revendiquée, parfois bafouée de la musique dans l'histoire de la rationalité du $\mathrm{xx}^{\mathrm{e}}$ siècle. Les discussions et prises de position tournent alors autour du formel, de l'informel, de la sérialité, du retour au matériau, ou au contraire de sa fétichisation. Les philosophes se veulent philosophes de la «nouvelle musique» comme Theodor Adorno, créant ainsi une tradition dont on n'a pas fini de mesurer la portée, encore aujourd'hui. Pierre Boulez lui-même ne s'est-il pas présenté dans ses débuts comme le continuateur d'une sorte de Bauhaus en faveur d'un formalisme postsériel répondant en somme à une philosophie de la musique héritière non seulement du mouvement analytique viennois en musique mais aussi de celui de l'empirisme logique transmis en France par son médiateur, ami de Moritz Schlick, Louis Rougier² ? Une place doit être encore faite aux réflexions du musicologue allemand Carl Dahlhaus en particulier sur le thème crucial de l'autonomie de la musique dite «absolue », sa filiation et son héritage auquel la philosophie de Wittgenstein n'est pas étrangère si l'on prend en compte la tonalité schopenhauerienne de son Tractatus, mais aussi le lègue romantique de l'autosuffisance du musical pur transmis à l'École de Schoenberg par Eduard Hanslick et son «formalisme» en musique. Enfin, la manière dont Wittgenstein «pense la musique et la société (ou la Culture)» mérite aussi d’être rapprochée de celle d'Adorno autant que contrastée avec elle dans la mesure où le rapport de la société à la musique est celui d'une réflexivité critique dont le négativisme dialectique ne rencontre aucun écho chez Wittgenstein. Enfin, l'immanentisme grammatical wittgensteinien auquel nous ramène sa philosophie de la psychologie oblige à reconsidérer la conception d'une «grammaire de l'harmonie» au sein de l'harmonie, les lois qui structurent de l'intérieur, et non en surplomb, le son et la construction de ses séquences de timbres, à l'écart de toute «méta-harmonie » chez Wittgenstein en comparaison avec l'harmonie sonore étendue à l'atonalité chez Schoenberg, et cela indépendamment de l'allergie de Wittgenstein à cette dernière sur le plan du goût.

Enfin, dans la mesure où la question nous plonge en pleine analogie et peut-être nous y laisse, que penser de la référence à la musique? Quel est exactement le statut de ce paradigme dont le fonctionnement ne doit jamais être que partiel? Et quelle relation d'expression possible nous réserve-t-il, lui qui est d'ordinaire tenu pour vecteur d'indicible, face au problème, délicat entre tous, de l'ineffabilité? S'il est vrai que l'œuvre d'art donne accès à ce qui se trouve de l'autre côté du dicible, quelle en est au juste l'énigmatique place dans une philosophie du langage qui évalue le sens du langage comme celui d'une phrase musicale à l'aune d'un critère d'articulation? N'est-il pas trop facile de
2. Voir par exemple Boulez (1963, p. 29), où le mot de "structure " réfère à Louis Rougier, médiateur du Cercle de Vienne en France dans les années 1930. 
3. Voir plus bas cette question du compositeur Helmut Lachenmann.

4. [ndlr] Les citations des ouvrages de Wittgenstein renvoient aux numéros de propositions indiqués. faire de l'ineffable le vrai refuge de l'art? À ce point, la dimension aspectuelle de la musique, la troisième, révèle sa richesse. On peut en effet penser, en s'attachant à l'aspect que bien que l'art manifeste par sa présence l'ineffabilité par excellence de ce qui échappe au langage humain, il y a bel et bien place pour une forme d'expressionnisme esthétique lié à la possibilité de décrire des réactions subjectives dans une dynamique aspectuelle. Cette direction, assez parlante, a l'avantage de souligner l'importance de la reconnaissance de l'expression (sans rapport avec un état de l'esprit) par analogie en particulier avec celle des traits du visage. Ce style de questionnement nous met sur une voie inédite qui contient aussi un défi à «l'argument du langage privé » selon lequel, comme il est bien connu, il n'est pas de règle qui soit susceptible d'être suivie en privé. Toutefois, si l'on nous accorde que les aspects échappent au privé, qui est le point de vue défendu ici (Soulez 2003a), on peut dès lors attendre de la musique des lumières sur l'articulation d'un complexe audible instantiant une forme de signification exprimable en première personne audelà de la saisie d'une Gestalt immédiate (Roger Scruton) (cf. Scruton, 2003). Se trouverait ainsi résolu le dilemme de l'aspect et de l'affect en musique 3 .

\section{La musique: un paradigme de style projectif en philosophie}

La musique apparait dans le Tractatus comme l'exemple par excellence de ce qui est au fondement inexplicable et irréductible de la représentatibilité de la forme logique en ce qu'elle projette l'un sur l'autre l'ensemble Monde et l'ensemble Langage, sans que l'on puisse justifier ce qui rend possible cette représentation dite logique. Les propositions peuvent représenter toute la réalité mais ne peuvent représenter ce qu'elles doivent avoir de commun avec elle pour pouvoir la représenter : à savoir la forme commune logique. (Il nous faudrait sortir du langage pour être capable de le faire, ce qui est impossible et folie) (cf. 4.12 $)^{4}$. [...]

Il est frappant que lorsque le Tractatus parle de musique, c'est à la partition qu'il nous renvoie comme à un système notationnel mis sur le même plan que des relations fonctionnelles. Les notes sur une portée fonctionnent comme des Bilder, des faits. Selon la référence à Hertz et à sa mécanique, dont on sait qu'elles ont inspiré à Wittgenstein ces mots : «Wir machen uns Bilder den Tatsachen» (2.1), il est donc également tentant d'associer les concepts ou Scheinbilder à des entités notales comme le fait d'ailleurs Ernst Mach dans un article de 1894 sur «La comparaison en physique». Ce qui entraîne encore à reconnaître que le son auquel correspond l'entité notale est pris dans une relation conventionnelle de symbolisant à symbolisé dotée d'une détermination univoque et excluant toute ambiguïté, par exemple l'ambiguiité qu'introduiraient les dissonances, la microtonalité, les bruits. 
Cependant, que dire concernant le plan de ce qui se projette? Wittgenstein parle comme Schoenberg d'Idée musicale, Musikalische Gedanke. On remarquera que l'Idée musicale se trouve sur le même plan que ce qui est projeté, le disque, la partition, la performance orchestrale, et même, ajoute Wittgenstein, les ondes sonores. Or, on se serait attendu à ce que l'Idée musicale occupe une place au sommet, sur le plan supérieur de la projection. Elle a donc le statut d'un représenté, issu de la projection, et possédant un caractère relationnel et construit.

Alors qu'est-ce que la musique? La $5^{\mathrm{e}}$ Symphonie elle-même? Wittgenstein ne répond pas à cette question. Il ne la pose pas davantage. Elle est « ce qui doit être pour que puisse être le cas» (5.5542), étant entendu que l'on ne demandera jamais en quoi consiste ce qui doit être, ici le devoir-être de la musique. Il faut partir de l'œuvre, ce qu'Adorno appelle «partir d'en bas». C'est le «cas» : cette partition que l'on lit, cette musique que l'on entend, cette performance à laquelle on assiste, le CD, c'est-à-dire, d'après 4.0141, l'ensemble ouvert des formes de projection, toutes projections isomorphes entre elles dans la mesure, bien sûr, où elles le sont de la même symphonie de Beethoven par exemple. Cette «Idée » peut être mise sur le même plan que l'Idée architecturale du «cône» de Roithamer dans le roman de Thomas Bernhard Korrektur (1975). Idée elle-même projetée d'achèvement par construction, nullement platonicienne, mais toute philosophique, la musique, comme l'architecture, est «ce qu'on ne peut anticiper que dans la mesure où cela se laisse construire ». L'Idée de cette concevabilité accomplie, pour «l'œuvre d'art d'une vie », dit encore le roman en désignant la sœur de Wittgenstein (Bernhard, 1975, trad. fr., p. 243), est bel et bien objet de construction doté d'une prose. Cette Idée ne s'approche pas, ne s'esquisse pas par défaut comme chez Platon à partir de traits partiels ou insuffisants. Elle est atteinte ou n'est pas du tout.

Il semblerait donc que la force exemplaire de la musique soit aussi de permettre de saisir par une sorte de Gleichnis l'indicibilité de l'Idée, étant entendu qu'on ne forcera pas cette indicibilité. Mais indicibles, les relations internes toutefois s'expriment. Il leur correspond dans les symboles des «traits » qui sont ceux de la réalité. Ces traits se laissent décrire. Ils ne sont donc pas synonymes d'ineffabilité absolue. De même, la musique montre comment se déploie ce qui, ne pouvant se dire, est doué d'auto-expressivité, et cela à l'aide de graphes. L'auto-monstration du sens articulé aussi bien que non verbal est donc censé être l'apanage de la musique d'après le Tractatus et il le faut bien pour que les analogies que nous avons mentionnées gardent toute leur portée.

On peut alors se demander si Adorno a raison de déclarer que la philosophie de Wittgenstein, comme tout positivisme instaurant une ligne de partage nette 
entre le sens dicible et le non-sens, condamne l'art à l'ineffabilité, car dans son esprit, si l'art dépasse le dicible, c'est qu'il échappe à l'expression articulée. Or, nous venons de montrer au contraire que le principe d'articulation est un principe qui transcende le langage et peut avoir une application plus large. Wittgenstein serait peut-être plus proche d'un Valéry qui, condamnant les "poètes-philosophes», renvoyait, par une vigoureuse «critique du contenu», l'art à un pur déploiement formel.

L'assimilation certes rapide, pour ne pas dire fautive de Wittgenstein au positivisme par Adorno, ne doit pas nous cacher un autre trait bien particulier, c'est que la musique illustre "la possibilité de toute Bildhaftigkeit», de toute figurabilité. Suivre Adorno en oubliant cette particularité, ce serait comme d'attribuer à Wittgenstein la thèse que la musique que l'on écrit ne s'entend pas. Rien n'est plus faux pour Wittgenstein qui, bien au contraire, confie à la musique le soin de faire entendre une prose qui se passe de tout support verbal. Cela veut dire que, en tant que "prose», la musique assure sa propre significabilité indépendamment des mots. Ce trait est propre à la conception de la musique dite «absolue » selon la tradition romantique de la musique «purement» instrumentale. Elle «se» dit donc, elle est sens plutôt qu'elle n'a un sens. Dans cette mesure, on peut parler de «signification musicale» comme le fait plus tard Suzanne Langer dans Philosophy in a New Key (Harvard University Press, 1956, $3^{\mathrm{e}}$ édition).

Si Wittgenstein n'avait pas implicitement adhéré à cette filiation de la musique absolue, il n'aurait pas pu faire de la musique le paradigme de la représentabilité logique en ce qu'elle illustre l'impossibilité d'une représentation de la forme commune entre le représentant et ce qu'il représente. Dans les propositions 4.014 et suivantes, l'«Idée musicale» (Tractatus), ou Musikalische Gedanke, ne se rapporte pas non plus à une représentation subjective. En disant Gedanke, qui est aussi le mot de Schoenberg, Wittgenstein introduit Frege dans le champ du musical. Gedanke n'est-il pas la pensée objective dont la logique s'occupe? C'est donc à une certaine logique que l'exemple de la musique est capable de nous renvoyer dans la même mesure où «elle vise le vrai », comme a dit Adorno après Schoenberg. Le thème, le motif, les variations et leurs développements, tout cela vient en effet servir l'Idée de manière à la fois constructive et expressive (en un sens non sentimental). Le mot «pensée » vient souligner le caractère fregéen d'autonomie objective qui revient aussi à la musique depuis Eduard Hanslick.

Est «contenu expressif», rappelle le musicologue allemand Carl Dahlhaus dans son Schoenberg (1997), ce qui résulte du « tracé gestuel » que laisse après elle 
particulières vis-à-vis du système qui en résulte. D'après lui, Schoenberg pense ici à Bach. Maintenant, il est crucial de comprendre que c'est cette même dépendance formelle instruisant un parcours d'objectivation, qui appelle à dépasser les schémas notationnels eux-mêmes, c'est-à-dire un certain formalisme. Il ne s'agit certes plus du «formalisme» au sens de Hanslick qui, au milieu du XIX ${ }^{e}$ siècle, est devenu le champion de l'antiwagnérisme et défendu l'idée que le contenu de la musique, ce sont des «formes sonores animées» («Tönend bewegte Formen», Du Beau dans la musique, 1854). Pour Dahlhaus, dans le cas de la musique, il s'agit évidemment des schémas notationnels de l'écriture musicale. L'œuvre se caractérise ainsi par une «prose» autonome de relations sonores. En tant que «prose», elle se suffit à elle-même, et n'a donc aucun besoin du secours d'un support textuel, récit ou histoire. Comme on l'a reconnu plus haut, «prose » veut au contraire dire que la musique se passe de mots. Lidée, venue du romantisme allemand, caractérise la musique purement instrumentale «déliée» de toute référence au "vers poétique» comme le souligne le double sens de «ungebundene Rede» dû à Johann Nikolaus Forkel, le grand biographe de Bach (Leipzig, 1802). Chez Schoenberg aussi, l'expression de «prose musicale» dénote un «langage musical délié» où les idées mélodiques sont fondées en elles-mêmes et nullement par rapport à une histoire. Sous le titre du premier chapitre de son Schoenberg «Prose musicale» (texte de 1964), Dahlhaus suggère ainsi de passer outre les schémas exactement comme Wittgenstein appelle le lecteur à le faire pour ses propositions du Tractatus, dans l'avant-dernière proposition 6. 54. On sait qu'à cet endroit, Wittgenstein invite en effet à passer pardessus les propositions (überwinden) en enlevant l'échelle que le lecteur a dû gravir pour le (Wittgenstein) comprendre. Il est intéressant de voir un musicologue (Dahlhaus, 1997, p. 36, et note sur Wittgenstein, p. 42) interpréter, en référence à la variation développée de Schoenberg ${ }^{5}$, et comme un dépassement des notations musicales, cette proposition parfois qualifiée de sceptique ${ }^{6}$. Ce que Cora Diamond appelle «le rejet de l'échelle » est l'opération qui débouche sur ce qui en résulte : «voir le monde tel qu'il est», et dans la musique, je dirais, l'harmonicité interne du monde sonore.

Comme dans l'œuvre du philosophe, la logique musicale se dédouble donc en logique du symbolisme pour des relations externes de combinaisons purement formelles; c'est le formalisme que l'on peut jeter par-dessus bord, comme l'échelle, et la Logique du système d'ensemble qui incorpore l'Idée dans la grande forme organique de l'œuvre, laquelle est, chez Schoenberg également, l'expression objectivée d'une sorte de «volonté d'art», «Kunstwollen» (Alois Riegl) 7 .

En résumé, Wittgenstein met en place un paradigme musical pour la sémantique de la projection dans le domaine de l'œuvre. La musique se dit, et ce
5. Cf. sur la variation développée, Schoenberg (1995).

6. Notamment par Fritz Mauthner qui mentionne le "retrait de l'échelle " de Sextus-Empiricus à la fin de son Beiträge zu einer Kritik der Sprache (1901-1923).

7. Voir Questions de style (1893), Riegl, 1992. 
8. Cf. note ci-dessus, la forme est " une organisation d'idées musicales intelligibles, logiquement articulées", Schoenberg (1975, trad. fr., p. 15-17). qu'elle exprime en se disant est intérieur à son expression elle-même. Elle exemplifie en tout cas le fait d'être comprise sans explication, ce qui ne veut pas dire qu'elle est ineffable. J'ai montré dans un article sur Schoenberg qu'à bien des égards, les termes dans lesquels Wittgenstein parle de la musique recoupent le formalisme de l'École de Vienne quoique Wittgenstein n'y ait jamais reconnu la musique qu'il aimait (Soulez, 2003b).

Sous la plume de Schoenberg, le mot «Idée» (cf. Schoenberg, 1975), associé à «style», avait une connotation fortement combinatoire. Tout en constituant «l'essentiel en art», elle est devenue synonyme de forme, non pas la forme opposée au contenu ni bien sûr la forme platonicienne, mais ce qui, exprimable en termes de fonction et relations, se laisse «construire» en une architecture de symboles caractérisée par la «compréhensibilité et la cohérence » des éléments par rapport à la totalité douée de sens qu'ils forment par leurs interconnexions ${ }^{8}$. La dimension de la forme liée au contenu sentimental, l'Affekt, en musique, dont on ne séparait pas la «suavité» toute mélodique de la musique, n'a plus sa place dans cette conception «expressive » où, comme le montre le texte des manuscrits Gedanke de 1923, Gedanke ne renvoie plus à «mélodie» mais s'applique à la totalité formelle de l'œuvre composée.

Qu'une œuvre musicale «modélise » l'Idée musicale en la projetant annonce par ailleurs un motif que Nelson Goodman exploitera dans Languages of Art (1968) pour en tirer la thèse qu'il n'y a pas d'art sans un principe de projectibilité mais aussi que, en vertu de ce principe, une œuvre d'art est un «fairemonde» pour ne pas dire une «version-monde». Plus la version-monde relève de l'art, plus son attache au monde se fait ténue. Autrement dit, dans l'art, la musique surtout, la version-monde cesse d'être une version $d u$ monde. Cela peut paraître problématique pour les sciences de la nature si elles procèdent à la manière de l'art comme le pense Goodman accusé pour cette raison d'irréaliste, mais pas du tout pour la musique.

En conférant ainsi à l'Idée musicale cette fonction d'analogon structural de la possibilité formelle du sens, Wittgenstein fait d'une pierre deux coups : il donne une place à l'entité ineffable sans tomber dans le mythe de l'ineffabilité puisqu'on ne peut, on l'a vu, anticiper que ce qui se laisse construire par ailleurs, il dote cet ineffable-là d'une capacité auto-expressive exemplaire car aucun art mieux que la musique n'en dispose à ce degré, en toute indépendance vis-à-vis du texte, comme d'un monde qui lui servirait de référence. Le paradigme logique de la «prose musicale» inspire ces pensées de la projection communes à Schoenberg et à Wittgenstein, mais aussi à Goodman, au point qu'il n'importe plus qu'il vienne de la musique à la philosophie ou à l'inverse de la philosophie à la musique. 
Avec la musique, nous sommes donc en pleine comparaison, mais quelle sorte de «comparaison», et en quoi serait-elle une «méthode»? Ce qui passe au premier plan, ce sont en effet des traits d'affinités entre registres hétérogènes réclamant une méthode rigoureuse d'évaluation de ces traits. Celle-ci va cependant bientôt quitter la méthode verticale de la projection pour adopter le plan horizontal d'une comparaison entre jeux de langage en puisant aux ressources d'une grammaire comparative d'expressions de la «compréhension» : comprendre une phrase du langage, c'est comme comprendre une phrase musicale. Nous entrons dans une thématique comparative propre à la deuxième philosophie de Wittgenstein.

Une conception compositionnelle de la philosophie comme Dichtung se dessine alors, à laquelle on voit Wittgenstein attacher une importance nouvelle dégagée de la question transcendantale de «ce qui rend possible l'invention (erfinden) de formes» (5.555), qui reçoit d'ailleurs, comme on sait, une réponse négative puisque ce n'est pas une question à poser. La formule présentée comme problématique n'est pas exactement abandonnée plus tard, autour de 1930, mais plutôt, dans un contexte visant le «progrès moderne » et la science qui y tend, modulée en régime horizontal de questionnement sur la possibilité de la construction de formes, de préférence à la méthode de ceux qui, en science, s'intéressent aux constructions de superstructures. Si c'est bien encore le «compositeur » des formes qui parle ${ }^{9}$, ce n'est plus en vue de la construction d'un système, mais dans le but de «nur dichten » qui revient en propre à la philosophie comme activité. La «prose musicale» n'est donc pas perdue de vue, mais affinée dans le sens horizontal d'une activité de comparaison conduisant à « exécuter des ressemblances et des différences », comme le rappelle Gilles Granger dans un article resté célèbre. Dans cette optique, nous nous tenons à une distance critique de la poésie ${ }^{10}$ tant il est vrai que traduire dichten par écrire de la poésie serait «poétiser » la philosophie, ce qui est profondément, selon moi, anti-wittgensteinien.

Soit dit en passant, il est possible que ce motif non poétique de la composition en philosophie ait fait son chemin jusqu'à Stanley Cavell. Dans Must We Mean What We Say? (1969), Wittgenstein est dit appelé à «jouer» de la philosophie comme on joue de la musique.

\section{Geste et compréhension}

La deuxième approche de la relation de la musique avec le langage est celle qui inspire le plus de commentateurs et amène même certains à parler de «philosophie musicale» chez Wittgenstein (par exemple Philippe De Lara, 2003). Elle est centrée sur un schème de compréhension très proche de ce que nous
9. Voir le Tractatus.

10. Je rappelle ici un problème de traduction (de Dichtung = poésie) que j'ai soulevé à plusieurs reprises, notamment encore dans Soulez (2003a). 
11. Ce point est développé, sans référence à Schoenberg, dans Gargani (2003). dit Valéry dans ses Cahiers. Mais ce schème est constructif. Aldo Gargani a vu quelques affinités cruciales entre la construction de la phrase au sens formel chez Wittgenstein et la construction de la phrase chez Schoenberg. Dans son article de 1986, qui a fait date, intitulé «Procédures constructives et techniques descriptives : Schoenberg/Wittgenstein » (Gargani, 1986), il montre que ce qui est commun aux deux est le passage d'une forme à une autre pour la construction de nouvelles configurations qu'une simple démonstration logique ne réussit pas à faire voir; ainsi, la preuve en mathématiques fait voir une figure harmonieuse, une organicité esthétique, plutôt qu'une déduction ${ }^{11}$. Une organicité esthétique se présente sous la forme d'une synopsis de traits, une configuration, ou mieux une «constellation», par opposition à la linéarité de l'inférence. L'idée évoque aussi une opposition benjaminienne qui a frappé Adorno.

La citation qui a fait couler tant d'encre est celle-ci, bien connue : «Comprendre une phrase du langage est comme comprendre une phrase musicale. » Le «est comme» est très important et ne doit être télescopé. On va le retrouver avec le thème de la résonance. Quelques Remarques mêlées maintenant bien connues, font état de cette similarité qui n’est évidemment pas une ressemblance visuelle, ni auditive, mais structurale. Rappelons quelques points importants à ce sujet.

Suivant l'idée du geste comme «mouvement orienté», dont une Remarque mêlée, rapprochant musique et architecture, fait état, ce qui constitue le schème de la compréhension d'une phrase est la réponse à ce geste venu de l'œuvre, communiqué depuis elle, en réaction à ce geste, à savoir une réplique compréhensive. Cela sonne comme une variante du principe du «Zweckmässigkeit ohne Zweck» de Kant. Le geste se maintient comme visible tout seul. Il est tension maintenue du moyen à l'état nu de communiquer un sens quand on a enlevé à cette communication sa chair expressive, dit Giorgio Agamben (1992, p. 9).

Mais s'arrêter au «gestural» ne suffit pas à produire la similarité de la proposition avec la musique. Le gestural se laisse trop aisément sublimer en tension ineffable. La critique wittgensteinienne de l'ostension, dont le doigt du sujet indique un objet au-delà du langage, dénonce la magie de la visée dans cette perspective de renvoi à quelque chose d'halluciné plutôt que réel. Ici encore, nous sommes invités une fois de plus à refuser les facilités de la thèse de l'ineffabilité dont la musique servirait confusément la cause, en faisant ressortir plus de traits différentiels encore. Les lecteurs de Wittgenstein aujourd'hui sont tous unanimes à s'attaquer au «mythe » de l'ineffabilité dont la musique serait le vecteur privilégié. Et c'est je pense à bon droit. L'obnubilation fixée sur le geste aboutirait à la même impasse. 
Le geste est une réponse-réaction à un geste. Un «document humain» plutôt qu'une flèche d'accès vers un objet à connaître. Acte en «résonance », il manifeste ce que me fait l'œuvre, indépendamment de ce qu'exprime celle-ci, et indépendamment de ce que je ressens moi-même; il est en rapport avec ce que me dit l'œuvre, sans renvoyer à un objet à l'extérieur d'elle-même.

À ce point, le monodrame Erwartung de Schoenberg collerait assez bien avec l'analyse de l'attente dans les Remarques philosophiques de Wittgenstein. Les procédures de structuration de la phrase sont analogues. Comme tel et dans l'interaction gestuelle, ce qui est important est le site, le lieu actif devenu opératoire de cette action-réaction.

En philosophie comme en musique, on opère avec des « contenus formels » en quelque sorte «à la main» sur le mode artisanal d'un faire ${ }^{12}$. Nietzsche a pressenti ce motif esthétique du «contenu formel », pour reprendre l'expression grangérienne, en déclarant que «ce qui est formel pour le commun des mortels est contenu pour l'artiste, et inversement du contenu ». Autrement dit, c'est une affaire de point de vue. On peut dès lors comprendre que la solidarité du comprendre et du créer oblige à nouer les deux en une seule détermination. Toutefois, un des premiers à avoir relié aussi étroitement forme et contenu au point d'en faire une seule et même chose, c'est justement Eduard Hanslick, l'historien de la musique viennois du milieu du XIXe que nous avons cité plus haut. Nous avons aussi rappelé comment, à l'encontre de l'esthétique du sentiment, il a introduit le formel dans la musique comme contenu. La source de ce que Gilles Granger appellera plus tard le «contenu formel » pourrait bien résider dans l'esthétique en étroit rapport avec le motif de l'autonomie de la signification musicale, avant de revêtir plus tard, chez Granger notamment, un sens épistémologique.

Il est étonnant, et rarement remarqué, que de l'idée ayant en effet germé au cœur d'une problématique de l'autonomie du musical - à savoir que la «forme» est «contenu» (Inhalt en musique) et le contenu forme - , on est finalement passé à deux positions philosophico-musicales certes symétriques mais, en un sens méthodologique, diamétralement opposées : l'une de nature dialectique, celle bien connue d'Adorno, et l'autre opératoire, qui est celle développée par Granger en analogie explicite avec les arts, comme il est particulièrement visible au dernier chapitre de Formes, opérations, objets (1994).

Hanslick demande: «le contenu de la symphonie en si bémol de Beethoven? Quelle en est la forme? Où commence celle-là? Où finit celui-ci?... On donnera le nom de contenu aux sons eux-mêmes, mais ils ont déjà reçu une forme... Et qu'appellera-t-on forme? Encore les sons; mais ils sont une forme déjà remplie. » «Le contenu n'est pas le sujet. » Ce sont les «thèmes servant de base à une
12. Dimension soulignée à juste titre par Allan Janik, en référence à Hertz, cf. "Wittgenstein et le paradigme de l'art " (2003). 
certaine architecture». Le contenu ne peut être compris comme sujet mais c'est «l'Idée musicale, ce que l'on entend concrètement à l'audition d'une œuvre» (Hanslick, 1854, trad. fr., p. 163-164).

Il ne faut pas attendre trop longtemps pour que finalement le contenu formel constitue le matériau. C'est alors que, partant d'une réévaluation de la thèse d'Adorno, la question recouverte un temps par l'objectivisme viennois se reposera à nouveau de savoir si le matériau est subjectif ou non, si «penser le matériau » est une affaire subjective et de quelle subjectivité il s'agit ou non.

Mais parler de geste ne suffit pas. L'allusion n'a de sens que si on la rapporte au «mouvement orienté » par lequel je fais mien le geste de l'œuvre, mouvement d'intégration, d'intériorisation absolument crucial qu'un Grec aurait appelé mimesis, ou assimilation. Mais la mimesis ici est inverse de ce que les Grecs nous ont appris. Car au lieu de m'assimiler à l'œuvre comme de bas en haut en partant de ma condition, c'est au contraire d'une intégration qu'il s'agit (projective par Eindruck ou «impressionnement»), et cela de haut en bas. Cette intégration d'une structure gestuelle de l'œuvre en moi qui me modifie, m’affecte en profondeur, présente quelques affinités intéressantes avec le procès que Michaël Polanyi appelle «subreption» dans son livre Tacit Knowledge et qu'il voit à l'œuvre tout particulièrement dans les arts et les techniques. Cette «appropriation » se fait de l'œuvre à moi qui m'approprie le geste de l'œuvre, en réaction à ce qu'elle me fait. Ainsi, je la «comprends» activement sans avoir à identifier des parties constituantes de sa forme, sans passer par conséquent par un quelconque procédé de connaissance. Dans ce texte-clef auquel je pense et qui à mon sens est resté incompris, à savoir celui des Remarques mêlées et daté de 1948 (trad. fr., 2002, p. 73; avec association à la danse, p. 69), Wittgenstein précise en effet de façon frappante que le geste de l'œuvre «s'insinue en moi» et que c'est ainsi que «je le fais mien».

On peut rapprocher cette intériorisation du geste de celle qui serait l'idéal de la musique informelle libérée du carcan de la forme et qui reste à écrire, de «faire sienne en quelque sorte passivement la tendance du matériau » comme le dit de son côté Adorno (1982, p. 337). La formule suggère un laisser-être du matériau qui s'oppose à sa rationalisation par volonté de maitrise. La maîtrise du matériau «Materialbeherrschung» (mot de Max Weber) le fait dégénérer en instrument d'une rationalité technique et aliénante tandis que de son côté la volonté tourne en symptôme. Elle le comprime en le faisant taire. Déplorant cette orientation positiviste chez le Schoenberg du dodécaphonisme - ce qu'il appelle le «fétichisme du matériau» -, Adorno appelle au contraire le compositeur à libérer l'altérité qui est en lui en faisant ressortir ou résonner comme il dit «la tendance naturelle des sons», tendance interne en réalité 
toute de tension non résolue. Wittgenstein lui aussi en appelle à faire entendre la «tendance naturelle», trop souvent comprimée et contrariée par excès de formalité. «Laisser être le langage », comme il dit, est bien l'attitude dédogmatisante qui s'impose pour permettre au matériau du langage de déployer ses possibilités interrelationnelles.

Cependant, on ne saurait pousser plus loin le parallélisme de ces exhortations d'Adorno et Wittgenstein à laisser être le langage comme «laisser être le matériau du son » rendu à sa pleine autonomie intrinsèque. Wittgenstein ne pense pas «matériau» à propos du langage, même s'il n'est pas loin de lui reconnaître une dimension morphologique tant il est vrai que proférer un mot, une phrase, c'est aussi, morphologiquement, faire entendre un son, un Klang.

Pour revenir à Adorno qui reproche à Schoenberg d'avoir valorisé à l'extrême, à une phase de sa pensée musicale, l'écriture protocolaire, c'est en effet à l'exploitation d'un «matériau désensibilisé » devenu «extérieur » chez Schoenberg qu'il s'en prend (voir Adorno, 1949). Dans son analyse, cela veut dire qu'il convient que le musicien se libère du carcan du «calcul sériel».

À mon sens, il n'est pas absurde de lire le même mouvement de libération auquel appelle Adorno dans cette thématique de la compréhension de la phrase-geste chez Wittgenstein : désublimiser les superstructures logiques, laisser être le langage comme dit Wittgenstein ${ }^{13}$, tout cela ne renvoie-t-il pas en effet à une forme comparable d'émancipation?

Je pense que oui, dans une certaine mesure seulement. En opposition au carcan du formalisme et dans le but d'une décrispation, Wittgenstein en effet a lui aussi, à sa façon, réclamé une attention privilégiée aux structures résonantes de la langue, à sa dimension de «coloration » (Färbung), liée au ton, à l'éclat sonore, aux manières de dire et de proférer, qu'il reproche à Frege d'avoir superbement ignorées en leur préférant une pensée objectifiée, arrêtée, un peu comme Aristote avait, il y a des siècles, reproché à Platon d'avoir immobilisé les Formes et ainsi rendu inexplicables l'action et la connaissance. Le parricide de Frege perpétré par Wittgenstein aboutirait ainsi à valoriser ce que le père avait écarté au seul profit de l'essence : la vie, la vie de la proposition. Frege ne voulait-il pas considérer le langage de la pensée, abstraction faite de sa couleur sonore associée à la subjectivité? Dans le paragraphe 16 des Fiches (1971), Wittgenstein nous rappelle en effet qu'il s'agit moins de saisir en quoi consiste ceci ou cela qu'elle «voudrait dire » que de voir les manières, les mimiques, le ton et le mode affectés pour le dire. Le renversement est complet de l'objet en la manière de le configurer par la coloration que l'usager du langage confère aux mots qu'il profère.

Ajoutons que, comme pour Valéry, et sans doute parce que le contenu est formel et la forme contentuelle, comprendre et créer sont bien deux attitudes
13. Dans une Dictée de 31 sur «notre méthode " (Wittgenstein 1997, vol. I, p. 139-145). 
14. Kraus n'hésite pas, et Benjamin après lui, à dénoncer "l'outrage fait aux mots" (par les journalistes! dit Kraus) dans le but de sauver "l'œuvre d'art " de la langue, une fois celle-ci libérée de "l'information " qu'on veut lui faire porter comme si la langue - et c'est une illusion à combattre - n'était qu'un moyen de communication.

15. Que l'espace réduit à un pur espace sonore est impossible. soudées l'une à l'autre dans l'acte compositionnel antérieurement à leur distinction analytique en forme et contenu.

«Laisser être le langage », dit Wittgenstein : l'analogie avec la musique projette ainsi une lumière intéressante sur la passivité d'un schème d'intériorisation qui renverse la maîtrise en être-maîtrisé. Il en est, dit encore Adorno, comme lorsque l'on dit «maîtriser la langue », on veut alors dire, précise-t-il, que c'est elle qui vous maitrise, parce qu'on la manie dans le strict respect des règles de justesse et de sens qu'elle contient. Il s'agit d'une soumission à la langue, d'une disposition propice à son écoute, en tendant vers elle, en quelque sorte, une «oreille acoustique» (Karl Kraus). Contre l'alliance de «la technique et du verbiage», renchérit Walter Benjamin dans son écrit de 1931 sur Kraus, « laisser être le langage », respecter le langage implique donc de restituer à celui-ci le «droit» de faire entendre son ordre intime en faisant entendre ses exigences éthiques de justesse pour un dire-droit. En réclamant une telle restitution, c'est un appel à la réparation qui se fait entendre comme pour « réparer le tort» fait au langage quand on le maltraite, en oubliant qu'il a les exigences de l'œuvre d'art ${ }^{14}$.

Cela va également dans le sens de l'Eindruck, du dehors vers le dedans, dans le sens de la «résonance » qui détermine le geste-réponse, la réplique gestuelle. C'est peut-être aussi cela que voulait dire Gilles Deleuze par ce phénomène de transmutation d'un « devenir sensible» en « univers sonore», par exemple «l'univers Debussy». Ainsi, tout devient son comme dans la fiction de Peter Strawson au deuxième chapitre de son livre Individus, à laquelle un philosophe kantien pourrait objecter ${ }^{15}$, mais certainement pas un compositeur. «Devenir son » est consubstantiel à la musique. Le «bloc sonore» consacre la sensation incorporée, objectifiée dans le matériau, comme asservie à lui. Assurément, on l'a compris, cette sorte de résonance n'est ni résonance de l'Être grec à l'origine du Logos (Heidegger) ni simple consonance agréable à l'oreille.

Cependant, pour ressaisir la manière dont il est possible de comprendre une phrase dans le langage comme l'on comprend une phrase musicale, poussons un peu plus loin l'analyse.

Dans les paragraphes 527 à 531 des Recherches philosophiques, Wittgenstein propose une grammaire comparée du «comprendre » dont il accentue l'aspect pragmatique. Venons-en donc à l'examen de ces remarques. Dans ces passages, il s'agit de la comparabilité des phrases du langage avec celles de la musique, mais l'air de famille entre des phrases au sein même de la musique est également éloquent à cet égard. La grammaire comparée du «comprendre » est un exercice grammatical de caractère, je dirais pluri-registre, servant à éclairer ce que c'est que «comprendre ». Elle propose des jeux en révélant chemin faisant que 
seul un autre jeu montre en quoi consiste le jeu précédent. La méthode est donc d'invention et de production d'autres parallèles autant pour avérer des traits d'affinité que pour dévoiler des différences. Elle nous apprend aussi qu'entre langage et musique, qui ne sont certes pas identiques en tous points, passe le même fil d'Ariane qui permet de saisir le lien entre comprendre ce qui est exprimé et exprimer ce qui est compris ${ }^{16}$, et cela toujours dans un jeu effectif. Ce fil d'Ariane n'est pas une forme logique commune qui serait sous-jacente à la comparaison, car il n'y a plus place, désormais, contrairement au Tractatus, pour des ressemblance de famille et aucune classe de FRP (Family Resemblance Predicates) n'est ici envisageable ${ }^{17}$.

Il s'agit d'un schème que j'appelle «esthético-pragmatique » où performer ce qui est entendu ne se distingue pas de comprendre, car la compréhension ellemême s'avère en acte. La compréhension est aussi peu la moitié de cet acte que la performance n'est la moitié de la compréhension. Cette solidarité lie les deux faces de ce qui constitue en réalité une seule et même action, au sens où l'on n'a pas un comprendre sans un jeu qui le montre, ni un jeu sans un comprendre. Elle les soude de telle façon qu'on n'a pas l'un sans l'autre, exactement comme dans le Tractatus, on n'a pas la structure logique du Spiegelbild sans son envers indicible seulement montrable. Elle a aussi quelques conséquences de poids sur le rapport d'intentionnalité entre comprendre et agir.

Pour revenir à ce schème esthético-pragmatique, je voudrais encore souligner qu'il donne corps au motif dont le sens ne serait que partiel s'il se limitait seulement à un trait de réception. Aux yeux de Wittgenstein, il n'y a désormais d'expressivité que moyennant ce passage à l'action consistant à performer ce qui est compris en le mettant en acte, acte qui à son tour produit des signes de reconnaissance par l'autre que j'ai effectivement compris. Le caractère esthétique lui-même se mesure à cette dimension d'efficience dans ce domaine où comprendre inclut un moment de «réaction » à ce que la chose entendue vous «fait». Ainsi, le moment de l'appropriation du geste de l'œuvre auquel je faisais allusion plus haut en référence à la Remarque mêlée de 1948 débouche-t-il ultimement sur l'action.

Il est curieux que Wittgenstein associe dans ces lignes l'expression ainsi comprise sous l'aspect de la physionomie du visage avec les variations dont, écrit-il, la vie est faite. La valeur «geste» de la musique demeure en dépit de la répétition d'un thème si le thème s'intègre dans la variation qui se poursuit en l'incorporant, jusqu'à engendrer de nouvelles formes. Cette précision est donnée dans une remarque antérieure datant de 1946.

Dans ce texte des Remarques mêlées, il est montré en quel sens le motif ne naît de rien d'invariant, préexistant à la mélodie, laquelle n'est elle-même rien
16. Sur cette articulation, cf. Wittgenstein (1997, paragraphes 157-158, 160-161 et 163 à 166).

17. Voir la très belle analyse de cette impossibilité de construire une classe de FRP (Family Resemblance Predicates) par Andrès Raggio (1969). 
18. Les exemples du lotus égyptien ou de la feuille d'acanthe que l'on retrouve plus tard sur des chapiteaux de colonnes grecques invitent à l'interprétation de "symboles" (les deux plumes surmontant la tête du dieu Amon par exemple).

19. Cf. à ce sujet le livre d'Alois Riegl, historien important de l'École d'histoire de l'art de Vienne, Stilfragen (Questions de style, 1893) ainsi que l'article de Henri Zerner sur cette école (1975). On lui doit en particulier l'idée de l'inscription dans le cadre d'une culture d'un motif, par exemple floral. Or, la notion d'ancrage culturel, quoique excluant toute typologie, a une certaine importance chez Wittgenstein. Le motif relève pour lui aussi d'un Denkstil ou "style de pensée".

20. Cf. Fiches (1971, § 159); voir surtout les paragraphes 992-997 des Remarks on Philosophy of Psychology (1980). de plus qu'une séquence de variation. La dimension temporelle de sa capacité à être rejouée dans un procès de «répétition », qui n'est jamais et ne peut être un procès de reproduction d'une entité identique à elle-même, est également décisive. C'est à ce point que, pour illustrer ce fait qu'entendre une mélodie, c'est saisir une ligne «avec un début, un milieu et une fin », Wittgenstein trace une arabesque, cette forme ondulée, un huit couché, caractéristique de la mélodie et qui a une si longue histoire.

Le cas de la répétition d'un motif se présente en particulier en histoire de l'art avec les formes ornementales récurrentes qui se développent à une époque donnée et dont on peut suivre les phénomènes de variations. Cependant, dans cette conception ornementale vue à travers l'histoire des formes, comme Alois Riegl invitait à le voir, c'est au contraire la monotonie du motif et la reproduction du pattern qui dominent ${ }^{18}$. Cependant, ici comme ailleurs, le propre du motif est que ce qu'il «signifie» n'est pas écrit sur lui. Il faut le replacer dans une série pour l'interpréter ${ }^{19}$ ou le resituer dans une «culture».

On peut montrer que, sans autres précisions terminologiques, c'est en usant librement du vocabulaire de son époque que Wittgenstein fait comprendre qu'un thème, loin d'être «écrit d'avance », est tout sauf un invariant préalablement posé, que le jeu ferait varier ensuite. Ce qui se passe en particulier en musique vaut pour la signification dans le langage. Pas de «germe» de sens que l'usage « développerait» comme une pelote que l'on dévide. Car rien d'invariant au départ ne varie ensuite.

Sous la plume de Wittgenstein, le propos hostile au programme pourrait viser le «motif» wagnérien comme l'on sait que Hanslick se plaisait à le faire environ 8 o ans plus tôt. Par contraste avec la «forme-programme » imputée par exemple au Ring, la variation est un processus de production de formes («Gestalten») toujours nouvelles, écrit-il. Il faut donc « répéter» lors même que rien ne se répète véritablement à l'identique, et répéter ici veut dire rejouer, faire entendre une nouvelle fois, non pas une chose identique à elle-même, mais précisément un «motif», à savoir quelque chose d'irreproductible, car irremplaçable, et qui constitue ultimement la limite de la comparabilité des phrases musicales (cf. Recherches philosophiques, $\$ 531$ ). Plutôt que de répéter quelque chose d'identique et de déjà là, Wittgenstein veut dire qu'entendre une mélodie «avec un début, un milieu et une fin» incite à la jouer de «manière correspondante»: «Vous avez à entendre l'air comme ceci; et vous le jouez de manière correspondante» (entsprechend spielen) ${ }^{20}$ dans le même moment (trad. française de während). Tel est bien le «signe de reconnaissance» que je l'ai «compris». Motif et variation sont donc inséparables. Ils progressent ensemble. L'un est par l'autre mais rien n'est inscrit dans le motif dont la variation serait la reproduction 
variée. S'il semble y avoir du même et de l'autre, c'est donc pure illusion. Il n'y a qu' «altération», Veränderung.

Ces lignes sont philosophiquement fertiles mais, sans en avoir l'air, elles sont fortement inspirées des propos qui devaient courir à l'époque sur la variation. À dire vrai, l'idée d'une variation d'un motif en tant que «répétition variée» circule parmi les musiciens. On la trouve à l'œuvre chez Anton Webern dans «Variations pour piano », opus 27, écrites en 1936. Bien que Wittgenstein ignorât superbement la musique sérielle, on peut faire l'hypothèse qu'il a intégré des points de discussion qui ont influencé sa réflexion sur le motif dans sa comparabilité avec le thème et la variation en musique. Le point où achoppe ultimement la comparabilité de deux phrases coïncide avec l'élément irreproductible dans le processus de répétition de ce qui fait de la variation effectivement une variation. C'est pourquoi dans la répétition peut opérer la surprise quand bien même j'entends mille fois le même air.

Une analyse d'un critique acerbe de l'autonomie du musical, Aaron Ridley, propose une lecture intéressante de cette grammaire comparative du comprendre dans les deux registres. Son livre The Philosophy of Music (2004), qui prend pour cible le «purisme musical» de Peter Kivy (1990), s'appuie de façon développée sur le fait que la musique tout spécialement est de fait réfractaire à la paraphrase et même réclame, dans sa manière propre d'être malgré cela comprise, un aspect indispensable de «non-paraphraseability» à laquelle se laisse comparer la «non-paraphraseability» dans l'usage des mots. Ce qui rapproche les deux registres est donc une impossibilité : celle de traduire la même phrase dans une autre. Cependant, cette impossibilité qui caractérise un certain type de saisie du sens n'est absolument pas à verser au compte d'une interprétation ineffabiliste d'un «sens important» (ni spéculatif ni tautologique et vide de sens).

Le passage sur lequel il fonde cette lecture est le début du paragraphe 531 des Recherches philosophiques : «Nous parlons de la compréhension d'une phrase au sens où la phrase peut être remplacée par une autre qui dit la même chose, mais aussi au sens où elle ne peut être remplacée par aucune autre.» Il est clair que la paraphrasibilité du contenu d'une phrase exclurait d'emblée tout «comprendre » : «If all understanding were merely understanding of paraphraseable content, after all, understanding itself could never begin » (op. cit., p. 30). C'est cela que veut dire la formule "une phrase dit ce qu'elle dit et rien d'autre», mais cela n'empêche en rien l'importance du «context-dependency» de cette phrase non rephrasable.

Par là, il est possible de comprendre que Wittgenstein maintient dans sa philosophie seconde l'autonomie du musical «en contexte » et cela sans contradiction malgré l'apparence. 
21. Ich denke an eine ganz kurze von nur zweite Takten. Du sagst : "Was liegt nicht alles in ihr!" Aber es ist nur, sozusagen, eine optische Täuschung, wenn du denkst, beim Hören gehe vor, was in ihr legt. ("Es kommt drauf an, wer's sagt") (Nur in dem Fluss der Gedanken und des Lebens haben die Worte Bedeutung).
Lisons le paragraphe 173 des Fiches :

Je pense à une phrase musicale très courte de seulement deux mesures. On dit : «Il y a un monde en elle.» Mais c'est pour ainsi dire une pure illusion d'optique que de croire qu'en l'entendant il se passe tout ce qu'il y a en elle. («Tout dépend qui le dit.») (Ce n'est que dans le flux des pensées et de la vie que les mots prennent une signification $)^{21}$.

Commentons librement cette remarque de Wittgenstein. D'abord en la situant au paragraphe 172, Wittgenstein a rappelé que «comprendre une phrase musicale c'est comprendre un langage ». Aux paragraphes suivants, il explicite l'illusion sur un mode négatif, «ce n'est pas cela... »; le «cela» («maintenant, jetzt, j'ai compris») renvoie d'après la suite du paragraphe 175 aux mots que je prononce en guise d'explication de ma compréhension, comme si dans ces mots se trouvait la clef de ce que le thème signifie. Wittgenstein explicite la compréhension que je peux en avoir en alléguant la «familiarité» avec un contexte, et en nous dissuadant de mettre dans «cela — - à savoir mes mots d'explication, mon vécu, ce qui se passe en moi d'après ce que je crois - un quelconque contenu de compréhension. Dans mes «mots», rien n'est «contenu». Ce qui compte est l'environnement, l'intonation, la mimique, la manière dont je dis ces mots. "C'est une erreur de croire que vouloir dire quelque chose consiste en ceci ou cela", écrit Wittgenstein dans le paragraphe 16 des Fiches (1971). Mes mots ne renferment en effet aucune signification susceptible d'éclairer ma compréhension. La grammaire n'est pas la «philologie» comme Wittgenstein le déclare ailleurs. En termes fregéens propres à scandaliser Frege, l'important est bien, comme nous l'avons signalé plus haut, au passage, la «Färbung», la coloration et non le contenu de sens.

Une phrase courte de seulement deux mesures (Takt = mesure de musique) serait par exemple : «La jeune fille et la mort» de Schubert, citée dans la fameuse Remarque mêlée de 1946 que nous avons mentionnée et où nous trouvons le dessin d'une arabesque, sorte de courbe en forme d'onde pour figurer la mélodie en question.

Il s'agit d'un thème ou motif, segment complet d'une mélodie ou Linie \$ 527 des Recherches philosophiques. Il faut entendre cette phrase, cette ligne mélodique avec ce tempo, ce rythme, en soulignant le démonstratif, non pour appeler à mettre un contenu dans cette mélodie, mais pour attirer l'attention sur la singularité de ce tempo, par contraste avec le tempo en général, ce rythme, par contraste avec le rythme, comme il est dit dans les Recherches philosophiques, ce nombre, et non le nombre $\$ 65-71$.

Rappelons au passage que c'est dans ces remarques dirigées contre la définition fregé-russellienne du concept, que Wittgenstein, prônant la méthode de 
comparaison à l'aide de «paradigmes » ou échantillons linguistiques, suggère de renoncer à la recherche d'une forme commune aux différents prédicats correspondants, et de travailler seulement avec des FRP et les réseaux qu'ils forment. Il dit tout cela avec un exemple qui semble venir tout droit de Nietzsche : ce qui nous intéresse est cette feuille avec ce vert-ci, et non la feuille en général. Le démonstratif singularisant et la manière dont je le profère appartiennent à ce contexte de nos jeux de langage qui font que je ne parle pas dans le vide en l'utilisant.

On est donc averti qu'il n'y a rien à comprendre dans une petite phrase. La phrase en question ne contient pas «tout» le monde qui s'y réfléchirait comme dans une partie. Pas de métonymie ici. Cette relation non métonymique du monde à la petite phrase n'est pas de tout à partie, mais autre. Elle est, comme je le soutiens, une relation de tout à aspects de ce tout, relation expressive, et non de compositionnalité (tout de parties) ni de réflexivité au sens critique d'Adorno. Enfin, «Il y a un monde en elle» est la traduction française d'une expression de la bouche de quelqu'un. «Monde» ne s'y trouve pas en allemand. Mais ce n'est pas ce qui se passe, le processus (vorgehen, Vorgang), dans la dite phrase qui constituerait, croit-on, la chose à comprendre. La tournure est négative.

\section{Conclusion}

Nous avons distingué deux manières de traiter la philosophie en analogie avec la musique, chacune présupposant une certaine méthodologie de mise en rapport d'affinités et de différences entre musique et philosophie. Méthodologie projective d'abord, puis identificatoire par assimilation au geste de l'œuvre et intériorisation, et enfin, résonatrice selon un modèle de propagation ou rayonnement d'effets aspectuels sur le clavier des possibilités de jeux de langage avec des concepts en tant qu'échantillons sonores, la comparaison de la musique avec la philosophie maintient la relation de comparabilité sans identité. Elle ne propose cependant ni de comprendre la philosophie dans les termes de la musique ni de comprendre la musique dans les termes de la philosophie. Elle met en relief des traits pluri-registre d'analogies partielles entre deux procédures de composition en focalisant l'attention sur la structure interne d'un milieu, Mittel, ici les sons, là les concepts. L'immanentisme de Wittgenstein veut que milieu et matériau soient ici identifiés. Elle invite enfin à expérimenter sur pièces qu'il est impossible de parler de certaines formes de détermination opératoire de contenus formels dans un domaine autrement qu'en renvoyant à ce qui se passe opératoirement dans un autre. 


\section{B I B L I O GRAPHIE}

Adorno, Teodor W. (1949), Philosophie der neuen Musik, Tübingen, Mohr; trad. fr., Philosophie de la nouvelle musique, Paris, Gallimard, 1962.

Adorno, Teodor W. (1982), «Vers une musique informelle (1961)», Quasi une Fantasia, Paris, Gallimard, p. 291-340.

Agamben, Giorgio (1992), «Le geste et la danse», Revue d'esthétique, n² 22, p. 9-12.

Bernhard, Thomas (1975), Korrektur : Roman, Francfort, Suhrkamp; trad. fr., Corrections, Paris, Gallimard, 1978 .

Boulez, Pierre (1963), Penser la musique aujourd'hui, Paris, Denoël Gonthier.

Cavell, Stanley (1969), Must We Mean What We Say? A book of essays, New York, Scribner ; rééd., Cambridge, Cambridge University press, 2002.

Dahlhaus, Carl (1978), Die Idee der absoluten Musik, Kassel-Bâle-Tours-Londres-Munich, Bärenreiter-Deutscher Taschenbuch Verlag; trad. fr., L’idée de la musique absolue, Genève, Contrechamps, 1997.

Dahlhaus, Carl (1997), Schoenberg, Genève, Contrechamps.

Gargani, Aldo Giorgio (1986), «Procédures constructives et techniques descriptives : Schoenberg/ Wittgenstein », Revue Sud, p. 74-121.

Gargani, Aldo Giorgio (2003), «Le paradigme esthétique dans l'analyse philosophique de Wittgenstein », Rue Descartes, $n^{\circ}$ 39, «Wittgenstein et le paradigme de l'art», A. Soulez (dir.), p. 56-68

Goodman, Nelson (1968), Languages of Art; an Approach to a Theory of Symbols, Indianapolis, Bobbs-Merrill, 1968; $2^{\mathrm{e}}$ éd., Indianapolis, Hackett; trad., fr., Langages de l'art : une approche de la théorie des symboles, Paris, Hachette, 2005.

Granger, Gilles-Gaston (1994), Formes, opérations, objets, Paris, Vrin.

HansLick, Eduard (1854), Vom Musikalisch-Schönen, Leipzig, Rudolph Weigel; rééd. Wiesbaden, Breitkopf und Härtel, 1980; trad. fr., Du beau dans la musique : essai de réforme de l'esthétique musicale, G. Pucher (dir.), introduction par J.-J. Nattiez, Paris, Christian Bourgois éditeur, 1986.

JANIK, Allan (2003), «Art, artisanat et méthode philosophique selon Wittgenstein », Rue Descartes, $n^{\circ}$ 39, «Wittgenstein et le paradigme de l'art», A. Soulez (dir.), p. 18-27.

KIVY, Peter (1990), Music Alone: Philosophical Reflections on the Purely Musical Experience, Ithaca, Cornell University Press.

Koder, Rudolf et Wittgenstein, Ludwig (2002), Wittgenstein und die Musik. Briefwechsel Ludwig Wittgenstein-Rudolf Koder, Martin Alber, Brian McGuiness et Monika Seekircher (dir.), Innsbruck, Haymon.

Lara, Philippe de (2003), «Wittgenstein : une philosophie musicale?», Rue Descartes, $\mathrm{n}^{\circ}$ 39, «Wittgenstein et le paradigme de l'art», A. Soulez (dir.), p. 41-55.

Mauthner, Fritz (1901-1923), Beiträge zu einer Kritik der Sprache, 3 vol., Stuttgart, Cotta.

RAGGiO, Andrès (1969), «"Family Resemblance Predicates”. Modalité et réductionnisme » Revue Internationale de philosophie, G. Granger (dir.), p. 339-355.

Ridley, Aaron (2004), The Philosophy of Music: Theme and Variations, Édimbourg, Edinburgh University Press.

RIEGL, Alois (1992), Questions de style : fondements d'une histoire de l'ornementation (1893), préface de Hubert Damisch, Paris, Hazan, 1992.

Schoenberg, Arnold (1975), Style and Idea, Selected Writings of Arnold Schoenberg, Leonard Stein (dir.), Londres, Faber and Faber ; trad. fr., Le Style et l'idée, Paris, Buchet/Chastel, 1977.

Schoenberg, Arnold (1995), The Musical Idea, and the Logic, Technique, and Art of its Presentation, Patricia Carpenter et Severine Neff (dir.), New York, Columbia University Press. 
SCruton, Roger (2003), «Wittgenstein et la compréhension musicale», Rue Descartes, nº 39, «Wittgenstein et le paradigme de l'art», A. Soulez (éd.), p. 69-80.

Solomos, Makis, Soulez, Antonia et VAGgione, Horacio (2003), Formel / Informel : musique, philosophie, Paris, L'Harmattan.

Soulez, Antonia (200za), Comment écrivent les philosophes? de Kant à Wittgenstein ou le style, Paris, Kimé.

SoulEz, Antonia (2003b), «Schoenberg, penseur de la forme: musique et philosophie», dans Solomos, Soulez et Vaggione, 2003, p. 119-153.

Wittgenstein, Ludwig (1971), Fiches, Gertrude Elizabeth Margaret Anscombe et Georg Henrik von Wright (dir.), Paris, Gallimard.

Wittgenstein, Ludwig (1922), Tractatus Logico-philosophicus, avec une introduction de Bertrand Russell, Londres, K. Paul.

Wittgenstein, Ludwig (1980), Remarks on the Philosophy of Psychology, Gertrude Elizabeth Margaret Anscombe et Georg Henrik von Wright (éd.), Chicago-Oxford, University of Chicago Press-Blackwell.

Wittgenstein, Ludwig (1997), Dictées de Wittgenstein à Friedrich Waismann et pour Moritz Schlick, 2 vol., Antonia Soulez (dir.), Paris, Presses universitaires de France.

Wittgenstein, Ludwig (2002), Remarques mêlées, Paris, Flammarion.

Zerner, Henri (1975), «L'histoire de l'art d'Aloïs Riegl : un formalisme tactique », Critique, nº 339340, p. 940-952. 